\title{
Introduction to the Virtual Issue: Measurement in Political Science
}

\author{
William G. Jacoby \\ Department of Political Science \\ Michigan State University \\ East Lansing, MI 48824 \\ email: jacoby@msu.edu
}

\begin{abstract}
Welcome to the Political Analysis Virtual Issue on Measurement! The purpose of this issue is to highlight some of the work on measurement issues that has appeared in the pages of $P A$ over the past 24 years. As we will see, there is ample evidence that political scientists have been attentive to the challenges, problems, and opportunities that arise when researchers employ quantitative representations of empirical phenomena. This is critically important because it is often assumed that the health of a scientific discipline can be evaluated by examining the effectiveness with which its central concepts are measured (Kuhn 1977). The twelve articles chosen for inclusion in this virtual issue are:
\end{abstract}

Green, Donald Philip. (1990) “The Effects of Measurement Error on Two-Stage, Least- Squares Estimates." Political Analysis 2: 57-74.

McAvoy, Gregory E. (1998) "Measurement Models for Time Series Analysis: Estimating Dynamic Linear Errors-inVariables Models." Political Analysis 7: 165-186.

Brady, Henry E. (1990) "Factor verus Ideal-Point Analysis of Candidate Thermometer Ratings." Political Analysis 2: $97-129$.

Van Schuur, Wijbrandt H. (2003) "Between the Guttman Scale and Parametric Item Response Theory." Political Analysis 11: 139-163.

Londregan, John. (1999) “Estimating Legislators’ Preferred Points.” Political Analysis 8: 35-56.

Poole, Keith T. (2000) "Nonparametric Unfolding of Binary Choice Data." Political Analysis 8: 211-237.

Herron, Michael C. (2000) “Cutpoint-Adjusted Interest Group Ratings.” Political Analysis 8: 346-366.

King, Gary and Jonathan Wand. (2007) "Comparing Incomparable Survey Responses: Evaluating and Selecting Anchoring Vignettes.” Political Analysis 15: 46-66.

Pemstein, Daniel; Stephen A. Meserve; James Melton. (2010) "Democratic Compromise: A Latent Variable Analysis of Ten Measures of Regime Type." Political Analysis 18: 426-449.

Selway, Joel Sawat. (2011) "The Measurement of Cross-cutting Cleavages and Other Multidimensional Cleavage Structures." Political Analysis 19: 48-65.

Jackman, Simon. (2001) "Multidimensional Analysis of Roll Call Data via Bayesian Simulation: Identification, Estimation, Inference, and Model Checking.” Political Analysis 9: 227-241.

Bafumi, Joseph; Andrew Gelman; David K. Park; Noah Kaplan. (2005) "Practical Issues in Implementing and Understanding Bayesian Ideal Point Estimation.” Political Analysis 13: 171-187.

In a statement that is undoubtedly a classic within scientific literature, S. S. Stevens (1946) defined measurement as the process of assigning numbers to objects in meaningful ways. Of course, humans have been measuring things - usually with little fanfare or introspection about the process - since time immemorial. But, for most of human history, the objects being measured usually involved directly observable physical quantities. It has really only been for the past century and a half, when scientists have turned their attention to phenomena that cannot be reduced to counting operations, that measurement has become a topic of intrinsic interest and attention. And, the eventual 
result has been the development and evolution of measurement theory as a set of scholarly concerns that transcends any specific subfield or substantive application.

One variant of measurement theory, a subfield of mathematics, typically employs set theory to determine the logical conditions under which it is possible to make valid numerical assignments to objects (Hand 2004). While this approach provides an important epistemological foundation, it is not generally applicable to everyday scientific research activities. Instead, scholars concerned with measurement in the social and behavioral sciences have been much more attentive to issues involving the interpretation of measurement as well as the operations that can be used to generate measured quantities in the first place (e.g., Coombs 1964; Young 1981; Jacoby 1999). The twelve contributions in this virtual issue are excellent representatives of this latter tradition.

Any attempt to order or categorize a set of independent scholarly works inevitably will require some degree of arbitrariness and subjective judgment. Despite that caveat, I really believe that the works in this virtual issue fall rather nicely into a coherent set of complementary subsets. We begin with two articles on measurement error. These pieces are distinctive because they consider the effects of error-laden data in circumstances where other methodological issues typically receive more attention, non-recursive linear models (Green 1990) and time series analysis (McAvoy 1998).

Next, we turn to data theoretic concerns about geometric representations of information contained within a data matrix. Here, Brady (1990) contrasts "dominance" and "proximity" representations of rating-scale data, while Van Schuur (2003) presents three different manifestations of the cumulative response model. The papers that follow next, by Londregan (1999) and Poole (2000), carry on the discussion by focusing on ideal point estimation from binary data. They ask whether it is possible to construct a spatial representation of observations and choice alternatives that reproduces accurately the information in a data matrix composed of dichotomous entries.

The attention shifts in the next four articles to issues of interpretation. Of these, the first two papers deal with situations where a single measurement scale "hides" substantively important differences in the phenomenon being measured. Herron (2000) examines how distortions in cutting points along the underlying (and presumably unobservable) dimension probably generate inaccurate rating scales as empirical representations of the dimension. King and Wand (2007) develop guidelines for "differential item functioning," or individual differences in the ways that survey respondents interpret rating scales.

The next two papers focus on measuring comparative concepts, effectively imposing systematic regularity across a set of heterogeneous units. Pemstein, Meserve, and Melton (2010) take a sophisticated approach in developing a multiple-item measure of democracy that not only locates societies along a dimension, but also assesses the degree of uncertainty in their positions. Selway (2011) measures important characteristics of social structure, addressing the difficult task of reducing inherently multidimensional phenomena to meaningful and comparable quantitative dimensions.

Last, but certainly not least, the virtual issue wraps up with two papers on Bayesian approaches to measurement. Jackman (2001) focuses on multidimensional models of roll call data, emphasizing continuity with the longstanding common factor model. Finally, Bafumi, Gelman, Park,and Kaplan (2005) address a series of practical issues in Bayesian estimation of ideal point models, and provide useful insights about conveying the results from such models.

All of us are comfortable with the notion of statistical models that provide representations of structural relationships between variables. But, modern social science also regards measurement as a model that pertains to each of the individual variables. Careful attention and rigorous approaches are just as important for the latter type of models, as they are for the former. Taken together, the entries in this virtual issue of Political Analysis confirm that political scientists recognize this important point, and are concerned with valid and accurate empirical representation of theoretically relevant concepts.

\title{
References
}

\author{
Coombs, Clyde H. (1964) A Theory of Data. New York: Wiley. \\ Hand, David J. (2004) Measurement Theory and Practice: The World Through Quantification. London: Arnold Publishers. \\ Jacoby, William G. (1999) "Levels of Measurement and Political Research: An Optimistic View." American Journal of Political Science 43: \\ 271-301. \\ Kuhn, Thomas S. (1977) The Essential Tension: Selected Studies in Scientific Tradition and Change. Chicago: University of Chicago Press.
}


Stevens, S. S. (1946) “On the Theory of Scales of Measurement." Science 103: 677-680.

Young, Forrest W. (1981) “The Quantitative Analysis of Qualitative Data.” Psychometrika 46: 357-388.

About the Author: William G. Jacoby is Professor of Political Science at Michigan State University. He just completed a term as Director of the Inter-university Consortium for Political and Social Research Summer Program in Quantitative Methods of Social Research. And, he is starting a term as Editor of the American Journal of Political Science. Professor Jacoby's areas of interest include mass political behavior (especially ideology and values as elements of public opinion) and quantitative methodology (especially scaling methods and statistical graphics). 